\title{
Serum saturated fatty acids containing triacylglycerols are better markers of insulin resistance than total serum triacylglycerol concentrations
}

\author{
A. Kotronen • V. R. Velagapudi $\cdot$ L. Yetukuri • \\ J. Westerbacka $\cdot$ R. Bergholm $\cdot$ K. Ekroos $\cdot$ \\ J. Makkonen • M.-R. Taskinen • M. Orešič • \\ H. Yki-Järvinen
}

Received: 23 October 2008 / Accepted: 14 January 2009/Published online: 13 February 2009

(C) Springer-Verlag 2009

\begin{abstract}
Aims/hypothesis The weak relationship between insulin resistance and total serum triacylglycerols (TGs) could be in part due to heterogeneity of TG molecules and their distribution within different lipoproteins. We determined concentrations of individual TGs and the fatty acid composition of serum and major lipoprotein particles and analysed how changes in different TGs and fatty acid composition are related to features of insulin resistance and abdominal obesity.

Methods We performed lipidomic analyses of all major lipoprotein fractions using two analytical platforms in 16 individuals, who exhibited a broad range of insulin sensitivity. Results We identified 45 different TGs in serum. Serum TGs containing saturated and monounsaturated fatty acids

A. Kotronen and V. R. Velagapudi contributed equally to this study.

M. Orešič and H. Yki-Järvinen are joint senior authors of this study.

Electronic supplementary material The online version of this article (doi:10.1007/s00125-009-1282-2) contains supplementary material, which is available to authorised users.

A. Kotronen · J. Westerbacka · R. Bergholm · J. Makkonen •

M.-R. Taskinen $\cdot$ H. Yki-Järvinen

Department of Medicine, Division of Diabetes,

Helsinki University Central Hospital,

Helsinki, Finland

A. Kotronen $\cdot$ R. Bergholm $\cdot$ J. Makkonen

Minerva Medical Research Institute,

Helsinki, Finland

V. R. Velagapudi $\cdot$ L. Yetukuri $\cdot$ K. Ekroos $\cdot$ M. Orešič $(\bowtie)$

VTT Technical Research Centre of Finland,

Tietotie 2, P.O. Box 1000, 02044 VTT Espoo, Finland

e-mail: matej.oresic@vtt.fi

were positively, while TGs containing essential linoleic acid (18:2 n-6) were negatively correlated with HOMA-IR. Specific serum TGs that correlated positively with HOMAIR were also significantly positively related to HOMA-IR when measured in very-low-density lipoproteins (VLDLs), intermediate-density lipoproteins (IDLs) and LDL, but not in HDL subfraction $2\left(\mathrm{HDL}_{2}\right)$ or $3\left(\mathrm{HDL}_{3}\right)$. Analyses of proportions of esterified fatty acids within lipoproteins revealed that palmitic acid (16:0) was positively related to HOMA-IR when measured in VLDL, IDL and LDL, but not in $\mathrm{HDL}_{2}$ or $\mathrm{HDL}_{3}$. Monounsaturated palmitoleic $(16: 1$ $n-7)$ and oleic (18:1 n-9) acids were positively related to HOMA-IR when measured in $\mathrm{HDL}_{2}$ and $\mathrm{HDL}_{3}$, but not in VLDL, IDL or LDL. Linoleic acid was negatively related to HOMA-IR in all lipoproteins.

Conclusions/interpretation Serum concentrations of specific TGs, such as TG(16:0/16:0/18:1) or TG(16:0/18:1/18:0), may be more precise markers of insulin resistance than total serum TG concentrations.

Keywords Insulin resistance $\cdot$ Lipidomics $\cdot$ Lipoproteins · Mass spectrometry $\cdot$ Monounsaturated fatty acids ·

Saturated fatty acids $\cdot$ Triacylglycerols

$\begin{array}{ll}\text { Abbreviations } \\ \mathrm{HDL}_{2} & \text { HDL subfraction } 2 \\ \mathrm{HDL}_{3} & \text { HDL subfraction } 3 \\ \text { IDL } & \text { Intermediate-density lipoprotein } \\ \text { TG } & \text { Triacylglycerol } \\ \text { UPLC/MS } & \text { Ultra-performance liquid chromatography } \\ & \text { coupled to MS } \\ \text { VLDL } & \text { Very-low-density lipoprotein }\end{array}$




\section{Introduction}

Serum lipid and lipoprotein abnormalities are inherent components of the insulin resistance syndrome [1, 2]. Insulin resistance in the liver is characterised by the inability of insulin to inhibit hepatic very-low-density lipoprotein (VLDL) production [3]. This leads to elevation of serum triacylglycerols (TGs) [4, 5] and a low HDLcholesterol concentration [6].

The relationship between fasting serum TGs and insulin resistance measured using the hyperinsulinaemic-euglycaemic clamp technique in 1,308 non-diabetic individuals was weak [7]. However, TGs consist of multiple different fatty acids and are found not only in VLDL but also in other lipoprotein particles [8]. A recent cross-sectional study indicated that the fatty acid composition of TG determined using GC is differently related to surrogate markers of insulin resistance [9]. The proportion of saturated TG 16:0 fatty acid was positively associated with fasting serum insulin concentrations and that of essential 18:3n-6 fatty acid negatively associated [9]. This raises the possibility that the specific increases in serum TGs containing saturated fatty acids and decreases in the TGs containing polyunsaturated fatty acids may characterise insulin resistance and thus weaken the association between insulin resistance and total serum TG concentrations. The relationships between individual serum TGs and insulin resistance have not been previously studied.

The type of fat in the diet is an important determinant of the fatty acid composition in serum [10, 11]. Dietary intervention studies have shown that an increased intake of saturated fat is associated with an increase in insulin resistance [12], whereas an increased intake of polyunsaturated fat has been shown to improve insulin sensitivity [13]. The majority of serum fatty acids are carried by lipoproteins [8], some of which are considered as proatherogenic while others as anti-atherogenic. Long-term prospective studies show decreased essential and increased saturated fatty acid concentrations in total serum to predict the metabolic syndrome [14] and cardiovascular disease [15]. However, it is unknown how fatty acid composition of individual lipoproteins relates to insulin sensitivity.

Lipoprotein particles have traditionally been analysed by measuring their total protein, phospholipid, cholesteryl ester and TG content [8]. Modern lipidomics techniques such as ultra-performance liquid chromatography coupled to MS (UPLC/MS) afford analysis of multiple abundant lipids (e.g. TG, cholesteryl esters, phosphatidylcholines) as well as bioactive lipid species (e.g. ceramides, plasmalogens and lysophosphatidylcholines) in parallel [16]. Although the bioactive lipid species represent a minor component of the lipid milieu, several lines of evidence suggest that they may play a role in, for example, inflammation [17] and antioxidative defence [18], which might contribute to atherogenity or cardioprotective properties of different lipoprotein particles. The relative abundance of reactive lipid species in different lipoproteins has not been previously examined in any species.

In the present study, we performed a comprehensive lipidomics analysis to determine concentrations of individual TGs and fatty acid composition in serum and major lipoprotein particles. We then analysed how changes in different TGs and fatty acid composition are related to features of insulin resistance and abdominal obesity. We also determined whether and in which lipoprotein particles bioactive lipids are present.

\section{Methods}

Participants and study design A total of 16 non-diabetic individuals were recruited by newspaper advertisements based on the following inclusion criteria: (1) age 1860 years; (2) no known acute or chronic disease based on history, physical examination and standard laboratory tests (blood counts, serum creatinine, thyroid-stimulating hormone and electrolyte concentrations) and ECG; and (3) alcohol consumption $<20 \mathrm{~g} /$ day. The study protocol was approved by the ethics committee of the Helsinki University Central Hospital, and each participant provided written informed consent. Blood samples were taken after an overnight fast for measurement of fasting plasma glucose, fasting serum insulin, $\mathrm{HbA}_{1 \mathrm{c}}$, serum lipids and lipoproteins as previously described $[19,20]$. Waist circumference was measured midway between spina iliaca superior and the lower rib margin, and hip circumference at the level of the greater trochanters [19]. BP was measured in the sitting position after 10-15 min of rest using a random-zero sphygmomanometer (Erka, Germany). None of the participants used oral contraception or hormonal replacement. One woman was postmenopausal. Clinical characteristics of study participants are listed in Table 1 .

Lipoprotein fractionation The lipoprotein fractions were separated by sequential flotation in an ultracentrifuge [21] (Beckman L-60; Beckman, Palo Alto, CA, USA) at $4^{\circ} \mathrm{C}$ by using a Ti 50.4 Beckman rotor. Fresh serum was overlayered with $0.16 \mathrm{~mol} / 1 \mathrm{NaCl}, 1 \mathrm{mmol} / 1 \mathrm{EDTA}$ (density= $1.006 \mathrm{~g} / \mathrm{ml}$ ). The VLDL, intermediate-density lipoprotein (IDL), LDL and HDL fractions 2 and $3\left(\mathrm{HDL}_{2}\right.$ and $\mathrm{HDL}_{3}$ ) were fractionated using a $\mathrm{KBr} / \mathrm{NaCl}$ gradient as previously described [20]. The normalisation based on total protein content was used to estimate the lipid content of an individual lipoprotein particle. Protein concentrations in lipoprotein fractions were measured by a modified Lowry assay method [22]. 
Table 1 Clinical characteristics of the study participants

\begin{tabular}{llc}
\hline Variable & Value & Range \\
\hline$n$ (women) & $16(14)$ & \\
HOMA-IR & $1.70(0.94$, & $0.38-4.86$ \\
& $3.18)$ & \\
Fasting serum insulin (pmol/l) & $63 \pm 10$ & $14-154$ \\
Fasting plasma glucose (mmol/l) & $5.0 \pm 0.6$ & $4.3-6.4$ \\
HbA $_{1 \mathrm{c}}(\%)$ & $5.5 \pm 0.3$ & $4.9-6.1$ \\
Age (years) & $46 \pm 5$ & $39-60$ \\
Weight (kg) & $84 \pm 20$ & $56-122$ \\
BMI (kg/m ${ }^{2}$ ) & $30.2 \pm 5.3$ & $20.8-37.7$ \\
Waist (cm) & $99 \pm 14$ & $75-124$ \\
Fasting serum TGs (mmol/l) & $1.25 \pm 0.51$ & $0.42-2.37$ \\
Fasting serum HDL-cholesterol & $1.77 \pm 0.33$ & $1.06-2.22$ \\
$\quad$ (mmol/1) & & \\
Fasting serum LDL-cholesterol & $2.71 \pm 0.80$ & $1.52-4.23$ \\
$\quad$ (mmol/l) & & \\
Systolic BP (mmHg) & $128 \pm 14$ & $108-164$ \\
Diastolic BP (mmHg) & $83 \pm 7$ & $70-98$ \\
\hline
\end{tabular}

Values are mean $\pm \mathrm{SD}$ or median $(25 \%, 75 \%)$

Lipidomic analyses UPLC/MS-based analysis was used for lipid profiling. The applied platform affords broad screening of multiple lipid classes, including TGs, cholesteryl esters, and major phospholipids, from total lipid extracts within a single sample run and has been previously described in detail [16]. The platform is not optimal for detection of some negatively charged phospholipids such as phosphatidylserines and phosphatidylinositols. The method does not cover glycosphingolipids or the low molecular mass reactive lipids such as eicosanoids and NEFAs.

In short, $10 \mu \mathrm{l}$ of an internal standard mixture and $10 \mu \mathrm{l}$ $\mathrm{NaCl}(0.9 \%)$ were added to $10 \mu \mathrm{l}$ of the sample. Lipids were extracted from the samples with $100 \mu \mathrm{l}$ chloroform: methanol (2:1, vol./vol.) solvent and the sample was homogenised with a glass rod. Lipid extracts were analysed on a Q-ToF Premier mass spectrometer (Waters, Milford, MA, USA) combined with an Acquity UPLC (Waters). The lipid profiling was carried out using positive ion mode. The data were collected at mass range of $m / z 300-1,200$ with scan duration of $0.2 \mathrm{~s}$.

The obtained data were converted into netCDF file format using Dbridge software from MassLynx (Waters). The converted data were processed using MZmine software version 0.60 [23]. All reported lipids were identified with tandem mass spectrometry (UPLC/MS/MS) in positive and if necessary negative ion mode. The lipid concentrations were calibrated with class-specific internal standards.

Fatty acid analyses Analyses of serum and lipoprotein fatty acids were performed by adding $20 \mu \mathrm{l}$ of internal standard (heptadecanetrienoate TG C17:0+NEFA C17:0, $500 \mathrm{mg} / \mathrm{l}$ ),
$200 \mu \mathrm{l}$ choloroform:methanol (2:1, vol./vol.) solvent and $20 \mu 10.15 \mathrm{~mol} / \mathrm{l} \mathrm{NaCl}$ to $50 \mu \mathrm{l}$ of the sample. The samples were prepared for $\mathrm{GC}$ analysis as previously described [24]. GC was carried out using an HP 5890 GC-FID System that was directly connected to a FID detector (Agilent Technologies, Waldbronn, Germany).

Other measurements Serum unbound insulin concentrations were measured with a time-resolved immunofluorometric assay (PerkinElmer, Waltham, MA, USA). Inter-assay CV was $<4 \%$ in the concentration range $40-720 \mathrm{pmol} / 1$. Cross-

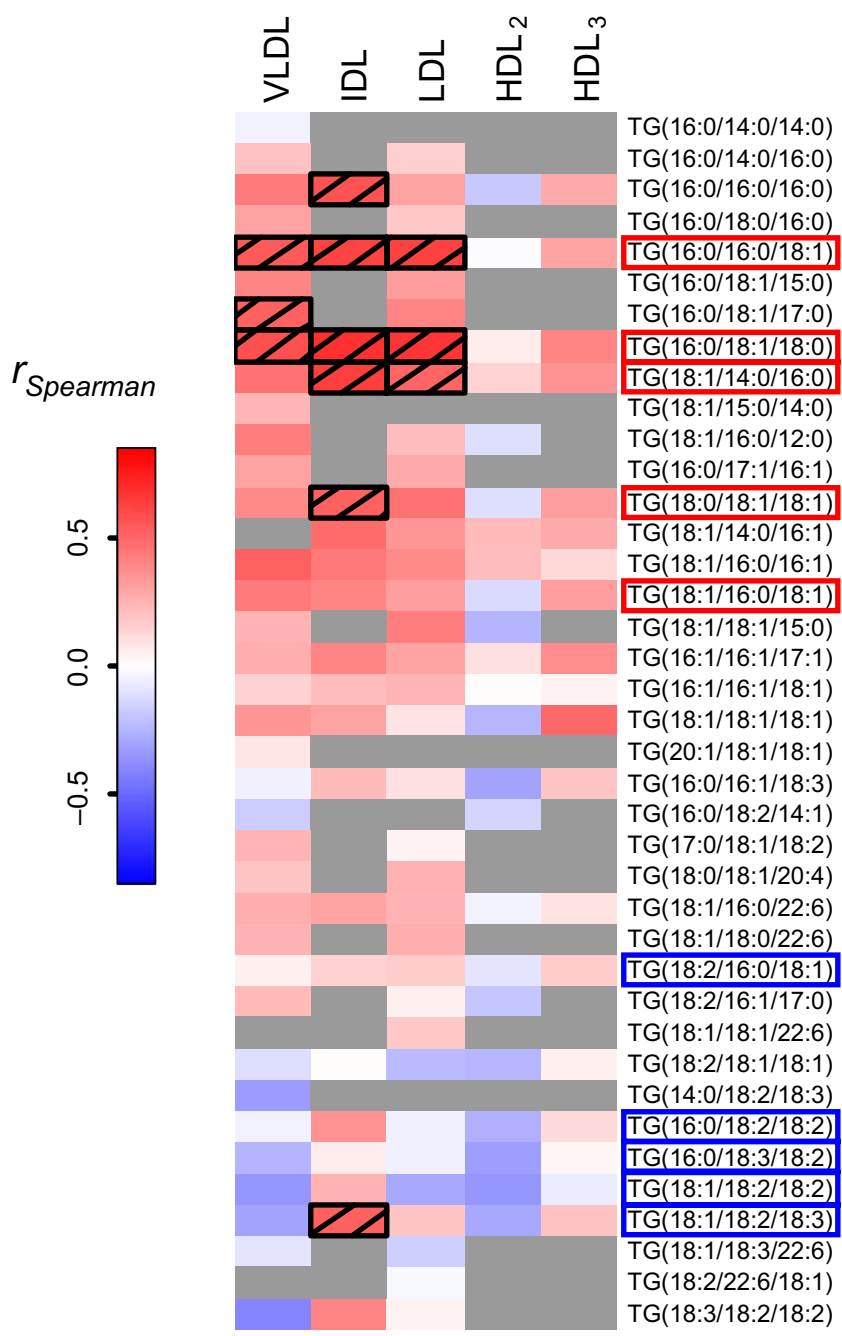

Fig. 1 Relationships between TG concentrations in different lipoprotein fractions and HOMA-IR. Spearman rank correlations were used to correlate the concentrations of each individual TGs $(\mu \mathrm{mol} / \mathrm{ml})$ within each lipoprotein fraction with HOMA-IR. The colour code corresponds to Spearman's correlation coefficient $\left(r_{\text {Spearman }}\right)$ values as shown. The lipids which were not detected within lipoproteins are marked in grey. Cross-hatching: $p<0.05$. The five serum TGs with the greatest positive (representing $29.4 \%$ of all serum TGs) and negative $(36.9 \%)$ weighted correlation coefficients are marked in red and blue, respectively 
Table 2 Relationships between esterified fatty acids and HOMA-IR of all the lipid species, expressed as molar \% in serum and its different lipoprotein fractions

\begin{tabular}{|c|c|c|c|c|c|c|}
\hline \multirow[t]{2}{*}{ Fatty acid } & \multicolumn{6}{|c|}{ Non-parametric correlation (Spearman) } \\
\hline & Serum & VLDL & IDL & LDL & $\mathrm{HDL}_{2}$ & $\mathrm{HDL}_{3}$ \\
\hline $14: 0$ & 0.45 & 0.37 & 0.09 & 0.11 & 0.27 & 0.37 \\
\hline $14: 1 n-9$ & 0.19 & $0.47 \dagger$ & -0.08 & -0.33 & -0.12 & -0.05 \\
\hline $16: 0$ & $0.56^{*}$ & $0.61^{*}$ & $0.59^{*}$ & 0.42 & 0.05 & 0.14 \\
\hline $16: 1 n-7$ & $0.73 * *$ & 0.13 & 0.11 & $0.46 \dagger$ & $0.68 * *$ & $0.49 \dagger$ \\
\hline $18: 0$ & 0.04 & $0.69 * *$ & $0.77 * * *$ & 0.11 & 0.12 & 0.08 \\
\hline $18: 1 n-9$ & $0.70^{* *}$ & 0.27 & 0.15 & 0.36 & $0.61 *$ & 0.42 \\
\hline $18: 1 n-7$ & 0.31 & 0.18 & 0.08 & -0.05 & 0.08 & -0.24 \\
\hline $18: 2 n-6$ & $-0.71 * *$ & $-0.72 * *$ & $-0.66^{* *}$ & $-0.62^{*}$ & $-0.62 * *$ & $-0.53 *$ \\
\hline $18: 3 n-6$ & 0.47 & $-0.50 *$ & $-0.48 \dagger$ & -0.03 & 0.26 & $0.43 \dagger$ \\
\hline $18: 3 n-3$ & -0.32 & $-0.52^{*}$ & $-0.57^{*}$ & $-0.52^{*}$ & 0.01 & -0.23 \\
\hline $20: 4 n-6$ & -0.14 & 0.24 & 0.42 & $0.45 \dagger$ & 0.14 & $0.44 \dagger$ \\
\hline $20: 5 n-3$ & -0.17 & 0.01 & 0.12 & 0.09 & 0.04 & 0.07 \\
\hline $22: 5 n-3$ & -0.33 & -0.22 & -0.04 & 0.19 & -0.05 & 0.16 \\
\hline $22: 6 n-3$ & -0.37 & -0.04 & -0.03 & -0.13 & -0.24 & -0.01 \\
\hline
\end{tabular}

${ }^{\dagger} p<0.1, * p<0.05, * * p<0.01, * * * p<0.001$

reaction with proinsulin and C-peptide was $\leq 0.1 \%$ [25]. HOMA for insulin resistance (HOMA-IR) was calculated using the formula of Matthews et al. [26].

Statistical methods Data are shown as means \pm SD. Correlation analyses were performed using Spearman's nonparametric rank correlation coefficient. The weighted correlation coefficient for each serum TG was calculated by multiplying the abundance (\%) of each serum TG by the Spearman's correlation coefficient. Five serum TGs with the greatest positive (representing $29.4 \%$ of all TGs) and negative $(36.9 \%)$ weighted correlation coefficients are shown in Fig. 1. The results remained essentially unchanged when men were excluded from the analyses, and when the concentrations of lipids $(\mu \mathrm{mol} / \mathrm{ml})$ were used instead of the protein-normalised values. Calculations were made using GraphPad Prism version 4.00 for Windows (GraphPad Software, San Diego, CA, USA), SysStat Statistical Package (SysStat version 10; SysStat, Evanston, IL, USA) and SPSS 14.0 for Windows (SPSS, Chicago, IL, USA). $p<0.05$ was considered statistically significant.

\section{Results}

Serum and lipoprotein TGS With the lipidomic approach, we identified 45 different TGs in serum. The most abundant serum TG molecules were TGs containing one palmitic (16:0) and two oleic (18:1) acids (TG[18:1/ $16: 0 / 18: 1], 19.9 \%$ of total) and $\mathrm{TG}(18: 2 / 16: 0 / 18: 1)$ (18.3\% of total). The Spearman's correlation coefficient for each individual serum TG and HOMA-IR were calculated.

Serum TGs with the greatest positive weighted correlation coefficient were significantly positively related to

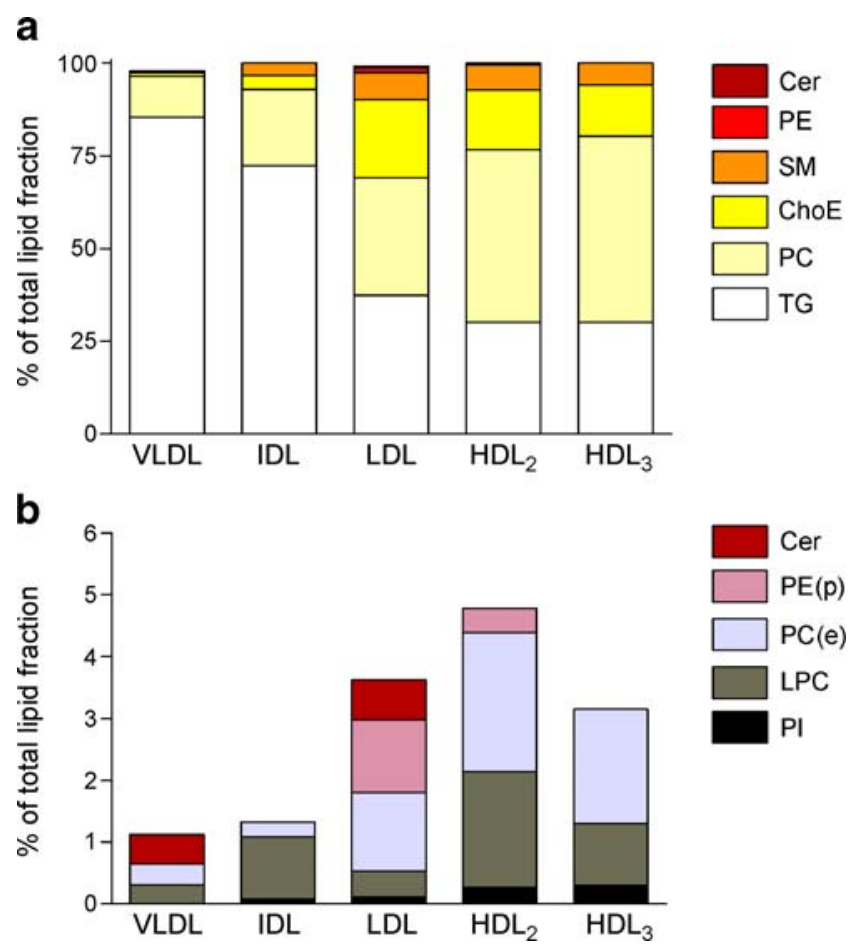

Fig. 2 Lipidomic characterisation of different lipoprotein fractions, based on per cent of the total lipid content within each fraction. Major (a) and minor (b) lipid classes are shown. Cer, ceramide; ChoE, cholesteryl ester; PC, phosphatidylcholine; PE, phosphatidylethanolamine; SM, sphingomyelin 


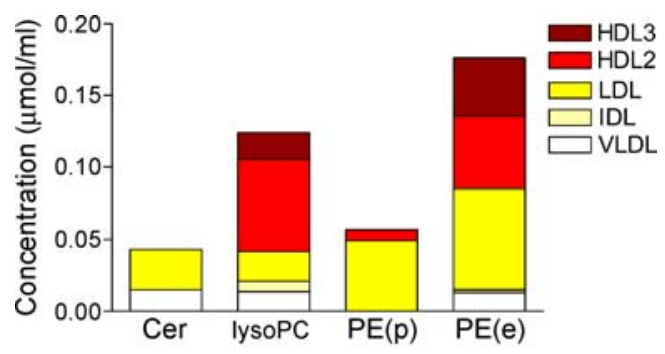

Fig. 3 Absolute $(\mu \mathrm{mol} / \mathrm{ml})$ concentrations of ceramides (Cer), lysophosphatidylcholines (lysoPC), ethanolamine plasmalogens (PE (p)) and ether-linked phosphatidylcholines $(\mathrm{PC}(\mathrm{e}))$ in lipoproteins

HOMA-IR when measured in VLDL, IDL and LDL, but not in $\mathrm{HDL}_{2}$ or $\mathrm{HDL}_{3}$ (Fig. 1). For the serum TGs that were negatively correlated with HOMA-IR, no clear trends were observed for the respective associations in different lipoprotein (Fig. 1). The results remain essentially unchanged if HOMA-IR was replaced by waist circumference (data not shown).

Serum and lipoprotein fatty acid composition Total serum esterified palmitic (16:0), palmitoleic (16:1 n-7), and oleic $(18: 1 n-9)$ acids were positively and linoleic acid (18:2 $n-6)$ negatively related to HOMA-IR. Analyses on total esterified fatty acids within lipoproteins revealed that palmitic acid was positively related to HOMA-IR when measured in VLDL, IDL and LDL, but not in $\mathrm{HDL}_{2}$ or $\mathrm{HDL}_{3}$. Linoleic acid was negatively related to HOMA-IR in all lipoproteins (Table 2). The distribution of esterified fatty acids across lipoproteins is shown in Electronic supplementary material (ESM) Fig. 1 and given in ESM Table 1.

Bioactive lipid species An overview of the lipid composition of lipoprotein fractions is shown in Fig. 2. Ceramides were found only in VLDL and LDL, whereas ethanolamine plasmalogens were found only in LDL and $\mathrm{HDL}_{2}$. Lysophosphatidylcholines and ether-linked phosphatidylcholines were present in all lipoproteins with the greatest abundance in $\mathrm{HDL}_{2}, \mathrm{HDL}_{3}$ and LDL. Concentrations of bioactive lipids in different lipoproteins are shown in Fig. 3 and given in ESM Table 2.

\section{Discussion}

Analyses on individual TGs in the present study revealed that serum TG molecules containing saturated and monounsaturated fatty acids, such as TG(16:0/16:0/18:1) and TG (16:0/18:1/18:0), correlated positively, whereas those containing essential fatty acids, such as TG(18:1/18:2/18:2), negatively with HOMA-IR and waist circumference. These
TGs correlated positively with features of insulin resistance only within VLDL, IDL and LDL, but not within HDL. Fatty acid analyses revealed that saturated 16:0 fatty acid (palmitate) was positively related to HOMA-IR only when measured in VLDL, IDL and LDL, whereas unsaturated 18:2 n-6 fatty acid (linoleate) was inversely related to HOMA-IR in all lipoproteins. In addition, we found that lipotoxic ceramides are present within VLDL and LDL in human serum.

We found that saturated, monounsaturated and polyunsaturated fatty acids containing TGs within the VLDLIDL-LDL axis and HDLs were differently related to insulin resistance. This might explain why the association between total serum TGs and insulin resistance is weak [7].

Saturated fatty acids containing TGs correlated positively with features of insulin resistance in VLDL, which is produced by the liver, and in IDL and LDL, which are derived from VLDL. The fatty acids in VLDL TGs have been shown to closely reflect sources of intrahepatocellular TGs [27]. Hepatic insulin resistance is associated with increased liver fat content even independently of obesity [28]. The fatty liver overproduces large TG-rich VLDL particles $[29,30]$. In individuals with a fatty liver, the contribution of TGs derived from de novo lipogenesis to both hepatic and VLDL TGs is significantly increased [27, 31]. Accelerated hepatic de novo lipogenesis leads to increased incorporation of palmitate $(16: 0)$, stearate (18:0), and oleate (18:1 n-9) into VLDL particles [32]. This in turn leads to decreased relative amounts of linoleate (18:2 n-6) in VLDL TGs [32]. In the present study, the significant positive relationships between features of insulin resistance and VLDL TGs containing saturated and monounsaturated fatty acids, such as TG(16:0/16:0/18:1) and TG (16:0/18:1/18:0), could therefore reflect increased hepatic de novo lipogenesis.

The analyses on esterified fatty acids of all lipids revealed that linoleic fatty acid decreases with increasing HOMA-IR and waist circumference in all lipoproteins. On the other hand, changes in saturated and monounsaturated fatty acids with respect to insulin resistance were lipoprotein-specific. Epidemiological prospective studies have shown that high proportions of esterified palmitic and stearic, and low proportions of esterified linoleic fatty acids in serum are risk factors for type 2 diabetes [33-35], myocardial infarction [36, 37], the metabolic syndrome [38] and overall and cardiovascular mortality [39]. The relationship between the amount of polyunsaturated fat in the diet and the respective proportions of serum esterified polyunsaturated fatty acids is strong $[40,10]$. The same relationships for saturated fatty acids are weaker [10, 41], and those between monounsaturated fatty acids non-existent $[10,41]$. These data would suggest that endogenous sources of saturated and monounsaturated fatty acids significantly 
contribute to serum and lipoprotein fatty acid concentrations, and that exogenous sources of fatty acids influence fatty acid composition in a similar manner in all lipoproteins.

Saturated esterified fatty acids were positively correlated with features of insulin resistance only in VLDL, IDL and LDL, and monounsaturated fatty acids only in HDL. The proportion of saturated fatty acids in serum lipid esters have been shown to be positively related to insulin sensitivity in some [42-44] but not all [45] studies. A recent study of 294 healthy 63-year-old Swedish men showed that correlation coefficients between HOMA-IR and plasma saturated fatty acids in phospholipids is 0.29 [44]. Both weak, but significant [43, 45], and insignificant [44] relationships between serum palmitoleic fatty acid and HOMA-IR have been reported. The specific pattern of the changes in lipoprotein fatty acid composition may explain the divergent results of the associations between features of insulin resistance and total serum fatty acid composition. Our results suggest that analyses of lipoprotein-specific rather than total serum fatty acid composition may more accurately reflect changes of fatty acids characteristic to insulin resistance.

Of the minor lipid sub-classes, proinflammatory and lipotoxic ceramides [17] were found within LDL and VLDL. These data in humans are novel and consistent with the ability of hepatocytes to synthesise ceramides de novo and incorporate into them into VLDL [46]. The absence of ceramides in IDL in the present study may be due to undetectably low concentrations of ceramides in IDL. Ethanolamine plasmalogens, known as endogenous antioxidants [18], were found only within LDL and $\mathrm{HDL}_{2}$ fractions. This may contribute to atherogenecity of low $\mathrm{HDL}_{2}$ [47].

Our results indicate that serum concentrations of specific TGs, such as $\mathrm{TG}(16: 0 / 16: 0 / 18: 1)$ or $\mathrm{TG}(16: 0 / 18: 1 / 18: 0)$, may be more precise markers of insulin resistance than total serum TG concentrations. Analyses of fatty acid composition in lipoproteins rather than total serum may help to define the role of saturated, monounsaturated and polyunsaturated fatty acids in the pathogenesis of the insulin resistance syndrome.

Acknowledgements This study was supported by research grants from the Academy of Finland (H. Yki-Järvinen), the Sigrid Juselius Foundation (H. Yki-Järvinen), the Jalmari and Rauha Ahokas Foundation (A. Kotronen), the Aarne and Aili Turunen Foundation (A. Kotronen), the Ida Montini Foundation (A. Kotronen), the Finnish Foundation for Cardiovascular Research (A. Kotronen), the Maud Kuistila's Memorial Foundation (A. Kotronen) and the Novo Nordisk Foundation (H. Yki-Järvinen). This work is part of the project Hepatic and Adipose Tissue and Functions in the Metabolic Syndrome (http:// www.hepadip.org), which is supported by the European Commission as an Integrated Project under the 6th Framework Programme (Contract LSHM-CT-2005-018734) (M. Orešič, H. Yki-Järvinen). We gratefully acknowledge M. Urjansson, K. Sohlo, H. Perttunen-
Nio, H. Hilden, I. Mattila, T. Seppänen-Laakso, A.-L. Ruskeepää, M. Katajamaa and J. Miettinen for excellent technical assistance, and the volunteers for their help.

Duality of interest The authors declare that there is no duality of interest associated with this manuscript.

\section{References}

1. Reaven GM, Hollenbeck C, Jeng CY, Wu MS, Chen YD (1988) Measurement of plasma glucose, free fatty acid, lactate, and insulin for $24 \mathrm{~h}$ in patients with NIDDM. Diabetes 37:1020-1024

2. Alberti KG, Zimmet P, Shaw J, IDF Epidemiology Task Force Consensus Group (2005) The metabolic syndrome-a new worldwide definition. Lancet 366:1059-1062

3. Kotronen A, Yki-Jarvinen H (2008) Fatty liver: a novel component of the metabolic syndrome. Arterioscler Thromb Vasc Biol 28:27-38

4. Malmstrom R, Packard CJ, Watson TD et al (1997) Metabolic basis of hypotriglyceridemic effects of insulin in normal men. Arterioscler Thromb Vasc Biol 17:1454-1464

5. Malmstrom R, Packard CJ, Caslake M et al (1997) Defective regulation of triglyceride metabolism by insulin in the liver in NIDDM. Diabetologia 40:454-462

6. Rashid S, Watanabe T, Sakaue T, Lewis GF (2003) Mechanisms of HDL lowering in insulin resistant, hypertriglyceridemic states: the combined effect of HDL triglyceride enrichment and elevated hepatic lipase activity. Clin Biochem 36:421-429

7. Ferrannini E, Balkau B, Coppack SW et al (2007) Insulin resistance, insulin response, and obesity as indicators of metabolic risk. J Clin Endocrinol Metab 92:2885-2892

8. Vance DE, Vance JE (2008) Biochemistry of lipids, lipoproteins and membranes, 5th edn. Elsevier, Hungary

9. Tremblay AJ, Despres JP, Piche ME et al (2004) Associations between the fatty acid content of triglyceride, visceral adipose tissue accumulation, and components of the insulin resistance syndrome. Metabolism 53:310-317

10. Ma J, Folsom AR, Shahar E, Eckfeldt JH (1995) Plasma fatty acid composition as an indicator of habitual dietary fat intake in middle-aged adults. The Atherosclerosis Risk in Communities (ARIC) Study Investigators. Am J Clin Nutr 62:564-571

11. Zock PL, Mensink RP, Harryvan J, de Vries JH, Katan MB (1997) Fatty acids in serum cholesteryl esters as quantitative biomarkers of dietary intake in humans. Am J Epidemiol 145:1114-1122

12. Vessby B, Unsitupa M, Hermansen K et al (2001) Substituting dietary saturated for monounsaturated fat impairs insulin sensitivity in healthy men and women: The KANWU Study. Diabetologia 44:312-319

13. Corpeleijn E, Feskens EJ, Jansen EH et al (2006) Improvements in glucose tolerance and insulin sensitivity after lifestyle intervention are related to changes in serum fatty acid profile and desaturase activities: the SLIM study. Diabetologia 49:2392-2401

14. Sjogren P, Basu S, Rosell M et al (2005) Measures of oxidized low-density lipoprotein and oxidative stress are not related and not elevated in otherwise healthy men with the metabolic syndrome. Arterioscler Thromb Vasc Biol 25:2580-2586

15. Wang L, Folsom AR, Eckfeldt JH (2003) Plasma fatty acid composition and incidence of coronary heart disease in middle aged adults: the Atherosclerosis Risk in Communities (ARIC) Study. Nutr Metab Cardiovasc Dis 13:256-266

16. Orešič M, Hänninen V, Vidal-Puig A (2008) Lipidomics: a new window to biomedical frontiers. Trends Biotechnol 26:647-652

17. Summers SA (2006) Ceramides in insulin resistance and lipotoxicity. Prog Lipid Res 45:42-72 
18. Zoeller RA, Grazia TJ, LaCamera P, Park J, Gaposchkin DP, Farber HW (2002) Increasing plasmalogen levels protects human endothelial cells during hypoxia. Am J Physiol Heart Circ Physiol 283:H671-H679

19. Kotronen A, Westerbacka J, Bergholm R, Pietilainen KH, YkiJarvinen H (2007) Liver fat in the metabolic syndrome. J Clin Endocrinol Metab 92:3490-3497

20. Taskinen MR, Kuusi T, Helve E, Nikkila EA, Yki-Jarvinen H (1988) Insulin therapy induces antiatherogenic changes of serum lipoproteins in noninsulin-dependent diabetes. Arteriosclerosis 8:168-177

21. Havel RJ, Eder HA, Bragdon JH (1955) The distribution and chemical composition of ultracentrifugally separated lipoproteins in human serum. J Clin Invest 34:1345-1353

22. Kashyap ML, Hynd BA, Robinson K (1980) A rapid and simple method for measurement of total protein in very low density lipoproteins by the Lowry assay. J Lipid Res 21:491-495

23. Katajamaa M, Miettinen J, Oresic M (2006) MZmine: toolbox for processing and visualization of mass spectrometry based molecular profile data. Bioinformatics 22:634-636

24. Seppanen-Laakso T, Laakso I, Hiltunen R (2002) Analysis of fatty acids by gas chromatography, and its relevance to research on health and nutrition. Anal Chim Acta 465:39-62

25. Toivonen E, Hemmila I, Marniemi J, Jorgensen PN, Zeuthen J, Lovgren T (1986) Two-site time-resolved immunofluorometric assay of human insulin. Clin Chem 32:637-640

26. Matthews DR, Hosker JP, Rudenski AS, Naylor BA, Treacher DF, Turner RC (1985) Homeostasis model assessment: insulin resistance and beta-cell function from fasting plasma glucose and insulin concentrations in man. Diabetologia 28:412-419

27. Donnelly KL, Smith CI, Schwarzenberg SJ, Jessurun J, Boldt MD, Parks EJ (2005) Sources of fatty acids stored in liver and secreted via lipoproteins in patients with nonalcoholic fatty liver disease. J Clin Invest 115:1343-1351

28. Seppala-Lindroos A, Vehkavaara S, Hakkinen AM et al (2002) Fat accumulation in the liver is associated with defects in insulin suppression of glucose production and serum free fatty acids independent of obesity in normal men. J Clin Endocrinol Metab 87:3023-3028

29. Adiels M, Taskinen MR, Packard C et al (2006) Overproduction of large VLDL particles is driven by increased liver fat content in man. Diabetologia 49:755-765

30. Fabbrini E, Mohammed BS, Magkos F, Korenblat KM, Patterson BW, Klein S (2008) Alterations in adipose tissue and hepatic lipid kinetics in obese men and women with nonalcoholic fatty liver disease. Gastroenterology 134:424-431

31. Timlin MT, Parks EJ (2005) Temporal pattern of de novo lipogenesis in the postprandial state in healthy men. Am J Clin Nutr 81:35-42

32. Aarsland A, Wolfe RR (1998) Hepatic secretion of VLDL fatty acids during stimulated lipogenesis in men. J Lipid Res 39:1280-1286

33. Hodge AM, English DR, O'Dea K et al (2007) Plasma phospholipid and dietary fatty acids as predictors of type 2 diabetes: interpreting the role of linoleic acid. Am J Clin Nutr 86:189-197

34. Wang L, Folsom AR, Zheng ZJ, Pankow JS, Eckfeldt JH, ARIC Study Investigators (2003) Plasma fatty acid composition and incidence of diabetes in middle-aged adults: the Atherosclerosis Risk in Communities (ARIC) Study. Am J Clin Nutr 78:91-98

35. Vessby B, Aro A, Skarfors E, Berglund L, Salminen I, Lithell H (1994) The risk to develop NIDDM is related to the fatty acid composition of the serum cholesterol esters. Diabetes 43:13531357

36. Ohrvall M, Berglund L, Salminen I, Lithell H, Aro A, Vessby B (1996) The serum cholesterol ester fatty acid composition but not the serum concentration of alpha tocopherol predicts the development of myocardial infarction in 50-year-old men: 19 years follow-up. Atherosclerosis 127:65-71

37. Miettinen TA, Naukkarinen V, Huttunen JK, Mattila S, Kumlin T (1982) Fatty-acid composition of serum lipids predicts myocardial infarction. BMJ 285:993-996

38. Warensjo E, Riserus U, Vessby B (2005) Fatty acid composition of serum lipids predicts the development of the metabolic syndrome in men. Diabetologia 48:1999-2005

39. Warensjo E, Sundstrom J, Vessby B, Cederholm T, Riserus U (2008) Markers of dietary fat quality and fatty acid desaturation as predictors of total and cardiovascular mortality: a populationbased prospective study. Am J Clin Nutr 88:203-209

40. Glatz JF, Soffers AE, Katan MB (1989) Fatty acid composition of serum cholesteryl esters and erythrocyte membranes as indicators of linoleic acid intake in man. Am J Clin Nutr 49:269-276

41. Nikkari T, Luukkainen P, Pietinen P, Puska P (1995) Fatty acid composition of serum lipid fractions in relation to gender and quality of dietary fat. Ann Med 27:491-498

42. Pelikanova T, Kazdova L, Chvojkova S, Base J (2001) Serum phospholipid fatty acid composition and insulin action in type 2 diabetic patients. Metabolism 50:1472-1478

43. Vessby B, Tengblad S, Lithell H (1994) Insulin sensitivity is related to the fatty acid composition of serum lipids and skeletal muscle phospholipids in 70-year-old men. Diabetologia 37:10441050

44. Gertow K, Rosell M, Sjogren P et al (2006) Fatty acid handling protein expression in adipose tissue, fatty acid composition of adipose tissue and serum, and markers of insulin resistance. Eur J Clin Nutr 60:1406-1413

45. Lovejoy JC, Champagne CM, Smith SR et al (2001) Relationship of dietary fat and serum cholesterol ester and phospholipid fatty acids to markers of insulin resistance in men and women with a range of glucose tolerance. Metabolism 50:86-92

46. Merrill AH Jr, Lingrell S, Wang E, Nikolova-Karakashian M, Vales TR, Vance de (1995) Sphingolipid biosynthesis de novo by rat hepatocytes in culture. Ceramide and sphingomyelin are associated with, but not required for, very low density lipoprotein secretion. J Biol Chem 270:13834-13841

47. Taskinen MR (2005) Type 2 diabetes as a lipid disorder. Curr Mol Med 5:297-308 